\title{
DIABETIC RETINOPATHY: IMPORTANCE OF PRIMARY CARE PHYSCIAN IN SCREENING
}

\author{
Naveen kumar $\mathbf{T}^{1^{*}}$, Nagi Reddy $\mathbf{T}^{2}$, Radha Kishan $\mathbf{N}^{3}$ \\ ${ }^{1}$ Department of Pharmacology, Apollo Institute of Medical Sciences and Research, Hyderabad, Andhra Pradesh, \\ India \\ ${ }^{2}$ Department of Ophthalmology, Melaka Manipal Medical college, Jalan Batu Hampar, Bukit Baru, Melaka, Malaysia \\ ${ }^{3}$ Department of Biochemistry, Apollo Institute of Medical Sciences and Research, Hyderabad, Andhra Pradesh, \\ India
}

Received 21 May 2015; Review Completed 13 June 2015; Accepted 19 June 2015, Available online 15 July 2015

\begin{abstract}
Objective: The objective of this study is importance of primary care physician in screening diabetic retinopahy patients Material and Methods : Patients of type-2 diabetes mellitus attending diabetic clinic in melaka manipal medical college, malaysia were prospectively reviewed. Risk factors associated in study population were assessed by biochemical parameters, clinical examination, retinal photographs and referred to ophthalmology clinic.

Results: The prevalence of non proliferative and proliferative diabetic retinopathy with type-2 diabetic patients was $17 \%$ and $1 \%$ with history of decreased in vision above age group of 50 years. In addition the risk factors in the study group associated with the development of diabetic retinopathy are uncontrolled HbA1c levels ( 27\%), high BMI (38\%), increased total cholesterol (9\%), uncontrolled fasting blood sugar levels (47\%), and increased duration of diabetes.

Conclusion: Diabetic retinopahy is highly prevalent in patients with type-2 diabetes mellitus. Our findings indicates primary care physicians are well educated in regards to diabetic eye disease and in identification of risk factors due to number of years experience.

Keywords: Proliferative and non proliferative diabetic retinopathy, risk factors, visual disability, type -2 diabetes mellitus
\end{abstract}

\section{INTRODUCTION}

Diabetic retinopathy is one of the most prevalent and leading cause of blindness among adult aged 20-74 years. Primary care physicians (PCPs) are front-line care givers for patients with diabetes and are most important in counselling the patients about proper treatment and complications regarding diabetic retinopathy. Therefore, understanding ocular manifestations of diabetes and knowing the screening guidelines can impact timeliness of referrals to ophthalmology by PCPs. ${ }^{1,2}$

Blindness caused by diabetic retinopathy is potentially preventable. Early detection and treatment of vision threatening changes in the retina can lead to a better visual out come. The primary care physician has access to all the diabetic population as the patients attend the primary care hospitals for their medical treatment. Ophthalmologists can provide specialized services like laser and vitrectomy. Regular screening and prompt referral by primary care physician can only prevent the blinding complication. ${ }^{3}$

\section{Pathophysiology}

Diabetic retinopathy is a micro vascular disease. It affects the capillaries of the retina and later the larger blood vessels are also affected.
Elevated blood glucose causes certain changes in the blood which in turn damage the vessel wall leading to various pathological lesions seen in the fundus picture. Several biochemical changes have been implicated which influence the disease process through changes in the cellular metabolism, signalling and growth factors. These include polyol accumulation, protein kinase activation, oxidative stress and accumulation of advanced glycation end products.

Alteration in the cellular components like increased agglutination of platelets leads to slowing of the blood stream. Protein kinase $\mathrm{C}$ activation leads to loss of pericytes, damage to the basement membrane and is responsible for the appearance of micro aneurysms which are a pouch like projections of the capillary wall. They are seen as pinpoint, red dot like lesions seen in the posterior pole of retina ${ }^{4}$ 
As the changes become longstanding endothelial cells are damaged resulting in disruption of blood retinal barrier. Leakage of large molecules like blood elements, proteins and lipids results in dot blot haemorrhages, retinal edema and thickening. The edema disappears leaving behind the hard exudates, which can be observed as yellowish well defined lesions often seen as clumps. These changes constitute non proliferative diabetic retinopathy and as long as macula is not involved the patient may not have any visual complaints although severe changes can occur in other parts of retina with normal vision. Similar changes occurring near the macula result in defective vision and are classified as diabetic maculopathy. ${ }^{5}$

\section{MATERIAL AND METHODS}

A population based case control study was done over a period of 1 year in melaka manipal medical college, malaysia patients attending the out-patient department with history of diabetes. Permission from institutional review board and written informed consent was obtained from subjects as per helsinki declaration.

300 patients, particiapated in the study group and all had type- 2 diabetes as defined by the absence of ketosis and adequate insulin reserve. Individuals aged less than 40 years and duration of diabetes less than 5 years, children less than 10 years were excluded from the study because of less risk of retinopahy. Age group 50 years and above were included in the study group.

\section{Clinical and biochemical parameters}

Anthropometric measurements including weight, height, and waist measurements were obtained using standardized techniques.

The body mass index (BMI) was calculated by formula: weight in kilograms divided by height in meters square. Blood pressure was recorded in the sitting position in the right arm to the nearest $2 \mathrm{mmHg}$ with a mercury sphygmomanometer.

Fasting capillary blood glucose was determined with a glucose meter (One Touch Basic; LifeScan, Johnson \& Johnson, Milpitas, CA) in all subjects after ensuring 8 hours of overnight fasting for estimation of plasma glucose and serum lipids with an autoanalyzer (Hitachi 912; Roche Diagnostics GmbH, Mannheim, Germany) using kits supplied by the manufacturer. Glycated haemoglobin (HbA1c) was measured by high pressure liquid chromatography (HPLC), using the variant machine (Bio-Rad, Hercules, CA).

\section{Ocular examination}

Visual acuity was recorded with an illuminated snellen chart and visual acuity was documented separately for each eye.

\section{Retinal studies}

Retinal examination was performed with direct ophthalmoscopy after dilating the pupils with 1 drop of phenylephrine $(5 \%)$ and tropicamide $(1 \%)$ in both eyes, until the best possible mydriasis was obtained.

The minimum criterion for diagnosis of diabetic retinopathy was the presence of at least one definite microaneurysm in any field photographed. Photographs were assessed and assigned a retinopathy level, and the final diagnosis for each patient was determined from the grading of the worse eye according to the ETDRS criteria for severity of disease in the individual eye. ${ }^{6}$

\section{RESULTS}

Of 300 cases known diabetic patients, 17\% had non proliferative retinopathy (NPDR), $1 \%$ had proliferative retinopathy with $33 \%<5$ years , $40 \%>5-10$ years, $20 \%>10$ years duration

Table 1: Prevalence of diabetic retinopathy in the study group

\begin{tabular}{|l|c|c|}
\hline & Number of cases & Percentage \\
\hline Nonproliferative retinopathy & 50 & $17 \%$ \\
\hline Proliferative retinopathy & 4 & $1 \%$ \\
\hline
\end{tabular}

Table 2: Risk factors associated in study population

\begin{tabular}{|l|c|c|}
\hline & Number of cases & Percentage \\
\hline Uncontrolled HbA1c & 80 & $27 \%$ \\
\hline Body Mass index(BMI) & 115 & $38 \%$ \\
\hline Increased total Cholesterol & 26 & $9 \%$ \\
\hline Uncontrolled fasting blood sugar & 140 & $47 \%$ \\
\hline Age (>50years) & 210 & $70 \%$ \\
\hline Duration of diabetes & 100 & $33 \%$ \\
\hline$<5$ years & 120 & $40 \%$ \\
\hline 5- 10 years & 60 & $20 \%$ \\
\hline$>$ 10years &
\end{tabular}




\section{DISCUSSION}

Diabetes and blindness caused by diabetic retinopathy is a major community health problem. The problem is ever increasing as the diabetic population and the duration of diabetes is increasing.

Modification of the associated risk factors as well as early detection and treatment of sight-threatening diabetic retinopathy can prevent blindness. Clinical practice guidelines recommend annual eye screening for patients with diabetes mellitus.

The major risk factors for diabetic retinopathy in this study were duration of diabetes and degree of glycemic control, consistent with findings in previous studies. ${ }^{7,89}$

The prevalence of non proliferative and proliferative diabetic retinopathy with type-2 diabetic patients (Table 1) was $17 \%$ (50 cases/300) and $1 \%$ (4 cases/300) from age 50 and above. In addition the risk factors in the study group (Table 2) associated with the development of diabetic retinopathy are uncontrolled HbAlc levels 27\% (80), high BMI 38\% (115) , increased total cholesterol 9\% (26) , uncontrolled fasting blood sugar levels $47 \%(140)$, age group above 50 years $70 \%$ (210), duration of diabetes

\section{REFERENCES}

1. Sinclair SH, Delvecchio C. The internist's role in managing diabetic retinopathy: Screening for early detection. Cleveland Clinic Journal of Medicine. 2004;71(2):151-59.

2. Paulus YM, Gariano RF. Diabetic retiopathy: A growing concern in an aging population. Properly diagnosing diabetic retinopathy requires a dilated fundus exam once or more per year. Geriatrics.2009;64(2):16-26.

3. Klein R, Klein BE, Moss SE. The Wisconsin epidemiological study of diabetic retinopathy: an Update. Aust NZ journal of ophthalmology. Feb 1990; 18(1):19-22.

4. Cunha-vaz J.G. Pathophysiology of diabetic retinopathy. Br J Ophthalmol .1978;62:351-55.

5. Scholl S,Kirchhof J, Augustin AJ. Pathophysiology of macular edema. Ophthalmologica . 2010; 224 suppl 1:8-15.
$<5$ years $33 \%(100), \quad 5-10$ years $40 \%(120)$ and $>10$ years $20 \%(60)$.

The strengths of this study are that it was based on retinal photography and standard grading techniques and the study included a large representative population.

\section{CONCLUSION}

Adherence to eye screening guidelines and the prompt referral of sight threatening diabetic retinopathy are essential in order to reduce the incidence of blindness among patients with diabetes mellitus. This emphasizes the need for routine retinal screening of diabetic individuals to detect diabetic retinopathy in the early stages.

Our findings reveal that regardless of the type of primary care training or the number of years of experience, primary care physicians are well educated in regards to diabetic eye disease. If our findings are generalizable to all primary care physicians, then the lack of examinations are likely due to an issue other than physician knowledge. Thus other avenues of investigation into the etiology of this problem such as examining physician compliance, patient communication, patient compliance or other barriers to healthcare need to be explored.

6. Moss SE, Meuer SM, Klein R, Hubbard LD, Brothers RJ, Klein BE. Are seven standard photographic fields necessary for classification of diabetic retinopathy? Invest Ophthalmol Vis Sci. 1989;30:823-8.

7. Varma R, Torres M, Pena F, Klein R, Azen SP. The Los Angeles Latino Eye Study Group. Prevalence of diabetic retinopathy in adult Latinos: the Los Angeles Latino eye study. Ophthalmology. 2004;111:1298-306.

8. Chen MS, Kao CS, Chang CJ, Wu TJ, Fu CC, Chen CJ. Prevalence and risk factors of diabetic retinopathy among noninsulin-dependent diabetic subjects. Am J Ophthalmol. 1992;114:723-30.

9. Broadbent DM, Scott JA, Vora JP, Harding SP. Prevalence of diabetic eye disease in an inner city population: the Liverpool diabetic eye study. Eye. 1999;13:160-5. 